\title{
Chordae Tendineae Rupture in the United States: Trends of Outcomes, Costs and Surgical Interventions
}

\author{
Elena V. Dolmatova, MD, ${ }^{* 1}$ Kasra Moazzami, MD, MPH, ${ }^{* 1}$ James Maher, $M D,{ }^{1,2}$ Marc Klapholz, MD, ${ }^{1,2}$ \\ Justin Sambol, MD ${ }^{3}$ Alfonso H. Waller, MD ${ }^{1,2}$ \\ ${ }^{1}$ Department of Medicine, Rutgers New Jersey Medical School, The State University of New Jersey, Newark, New Jersey; ${ }^{2}$ Division \\ of Cardiology, Department of Medicine, Rutgers New Jersey Medical School, The State University of New Jersey, Newark, New \\ Jersey; ${ }^{3}$ Division of Cardiothoracic Surgery, Department of Surgery, Rutgers New Jersey Medical School, The State University of \\ New Jersey, Newark, New Jersey
}

\section{ABSTRACT}

Objectives: To describe national trends in the incidence and outcomes of patients with chordae tendineae rupture (CTR).

Methods: Patients who were diagnosed with CTR between 2000 and 2012 were identified in National (Nationwide) Inpatient Sample (NIS) registry. CTR was defined using validated International Classification of Diseases, 9th Edition, Clinical Modification diagnosis (ICD9-CM) codes.

Results: A total of 37,287 (14,833 mitral valve repair, 7780 mitral valve replacement) CTR cases were identified. Overall, in-hospital mortality in CTR decreased by 3\% from 2000 to $2012(P<0.001)$. From 2000 to 2012 , the rate of mitral valve repair increased from $27.2 \%$ to $46.4 \%,(P<0.001)$ with a concurrent decrease in the rate of mitral valve replacement (from 27.8 to $17.7 \%, P<0.001)$. After multivariate adjustment, patient age $(\mathrm{OR}=1.04,95 \% \mathrm{CI} 1.03-1.06, P<0.001)$, congestive heart failure $(\mathrm{CHF})(\mathrm{OR}=2.08,95 \% \mathrm{CI} 1.19-3.64, P=$ $0.01)$, myocardial infarction $(\mathrm{MI})(\mathrm{OR}=3.58,95 \%$ CI $2.10-$ $6.11, P<0.001)$, Deyo/Charlson comorbidity index $(\mathrm{OR}=1.23$, $95 \%$ CI $1.07-1.41, P<0.003)$ and use of the intra aortic balloon pump $(\mathrm{IABP})(\mathrm{OR}=4.8195 \%$ CI $2.71-8.55, P<0.001)$ were found to be independently associated with greater odds of mortality in these patients. Additionally, mitral valve replacement was significantly associated with higher costs of hospitalization (coefficient 15693, 95\% CI 12638-18749, $P<0.001$ )

Conclusion: Mitral valve repair is associated with reduced inpatient mortality and costs compared with mitral valve replacement. A substantial increase in the percentage of cases undergoing mitral valve repair with a concurrent decrease in cases undergoing mitral valve replacement were observed. Increasing age and comorbidity index, history of $\mathrm{CHF}$ and $\mathrm{MI}$, and use of IABP were identified as factors that could increase the risk of mortality in patients with CTR.

\footnotetext{
*These authors contributed equally to this work.

Received fune 15, 2016; accepted December 28, 2016.

Correspondence: Alfonso H. Waller, MD, FACC, Division of Cardiology, Department of Medicine, Rutgers New Fersey Medical School, Rutgers, The State University of New fersey, 185 South Orange Avenue, Newark, N7 07103, United States; 973-972-4731; Fax: 973-972-2116; (Email: abw22@njms.rutgers.edu).
}

\section{INTRODUCTION}

Chordae tendinae rupture and papillary muscle rupture comprise two causes of acute mitral valve regurgitation, which can lead to acute pulmonary edema and cardiogenic shock.

Since the underlying problem of acute severe mitral regurgitation is mechanical, treatment options are limited to surgical repair or replacement, which may include pre-surgical mechanical support via a left ventricular assist device (LVAD) as a bridge to surgery, or intra aortic balloon pump (IABP) placement for reduction of pre- and after-load. Mitral valve repair (MVP) is thought to be superior to mitral valve replacement (MVR) for all etiologies of mitral regurgitation and is the most common procedure performed for mitral regurgitation in North America [Gammie 2009].

Given the rarity of the condition, only a handful of retrospective studies are available describing patient populations with chordae tendineae rupture (CTR) [Gabbay 2010] with even fewer studies focusing on treatment modalities for these patients [Kazui 2004; Rankin 2004]. Trends in mortality, independent predictors of mortality, shifts in patient population characteristics over time, and trends in management of CTR help physicians identify patients at risk and guide management accordingly.

In this study we utilized data from the National (Nationwide) Inpatient Sample (NIS) to describe the trends in mortality, comorbidities, costs, and MVP and MVR in patients with ruptured chordae tendineae, and determine predictors of mortality in this patient population in the US between 2000 and 2012.

\section{METHODS}

\section{Data Source}

The NIS database was used in the present study to collect data from 2000 to 2012. The database is robust and is the largest all-payer inpatient database representing approximately $20 \%$ of all admission to nonfederal and non-rehabilitation hospitals in the US [Steiner 2002]. Using the sampling weights provided in the database, national estimates could also be predicted.

\section{Study Design and Coborts}

The International Classification of Diseases, 9th RevisionClinical Modification (ICD-9-CM) diagnostic code 429.5 was used to identify all patients with ruptured chordae tendineae 
from 2000 to 2012. Primary procedure codes of 35.23 and 35.24 were also used to identify patients who underwent mitral valve replacement, and code 35.12 for mitral valve repair, respectively. All demographic data, including age, sex, race, insurance status, primary and secondary procedures, hospitalization outcome, and length of stay were queried using the database.

The comorbidities associated with patients with ruptured chordae tendineae were identified using the Agency for Healthcare Research and Quality (AHRQ) comorbidity measures. These measures use ICD-9-CM diagnoses in order to identify different comorbidities on the discharge date. The Charlson Comorbidity Index (CCI) was used to evaluate the severity of comorbidities [Deyo 1992]. This index contains 17 comorbid conditions with differential weights with a total score ranging from 0 to 33 . Higher CCI scores correspond to greater burden of comorbid diseases.

Inpatient costs of care were calculated by merging data from the NIS database with cost-to-charge ratios available from the Healthcare Cost and Utilization Project. The database provides total charges for each inpatient stay.
Costs could then be calculated by multiplying the total hospital charge with cost-to-charge ratios which are used to account for the inherent variability among hospitals and regions for any given procedure. All costs were standardized by adjustments for inflation according to the latest consumer price index data released by the US government on January 16, 2015.

\section{Statistical analysis}

National estimates were generated using hospital-level discharge weights provided by the NIS. The Chi Square test was used for categorical variables while continuous variables were compared using the Wilcoxon signed rank test. For analysis of trends, the Cochrane Armitage test was used for proportions and for continuous variables, the nonparametric test for trend by Cuzick was used. Two level hierarchical models (with patient level factors nested within hospital level factors) were created using the unique hospital identification number incorporated as random effects within the model. Patient level variables such as age, sex, Deyo modification

Table 1. Baseline Characteristics of Patients (2000- 2012)

\begin{tabular}{|c|c|c|c|c|c|c|}
\hline Female (\%) & $13,123(35.2)$ & $4039(27.2)$ & $2797(35.9)$ & $6397(42.8)$ & $<0.001$ & $<0.001$ \\
\hline Died (\%) & $1839(4.9)$ & $284(1.9)$ & $525(6.8)$ & $1076(7.2)$ & $<0.001$ & $<0.001$ \\
\hline White Race (\%) & $24,209(64.9)$ & $9748(65.7)$ & $5007(64.4)$ & $9556(64.5)$ & 0.051 & $<0.001$ \\
\hline $\mathrm{CHF}(\%)$ & $2,019(5.4)$ & $110(0.7)$ & $151(1.9)$ & $1763(11.8)$ & $<0.001$ & $<0.001$ \\
\hline Diabetes Mellitus (\%) & $3,283(8.8)$ & $994(6.7)$ & $598(7.7)$ & $1705(11.4)$ & 0.006 & $<0.001$ \\
\hline Hypertension (\%) & $14,901(40)$ & $6395(43.1)$ & $2504(32.2)$ & $6130(41)$ & $<0.001$ & $<0.001$ \\
\hline Peripheral vascular disease (\%) & $1880(5)$ & $629(4.2)$ & $345(4.4)$ & $917(6.1)$ & 0.49 & $<0.001$ \\
\hline Chronic Valvular Disease (\%) & $2,995(8)$ & $135(0.9)$ & $137(1.8)$ & $2728(18.2)$ & $<0.001$ & $<0.001$ \\
\hline Atrial Fibrillation (\%) & $15713(42.1)$ & $6895(46.5)$ & $3750(48.2)$ & $5189(34.7)$ & 0.014 & $<0.001$ \\
\hline Myocardial Infarction (\%) & $2,346(6.3)$ & $351(2.4)$ & $652(8.4)$ & $1372(9.2)$ & $<0.001$ & $<0.001$ \\
\hline Mean Deyo \pm SD & $1.28 \pm 1.37$ & $0.93 \pm 1.13$ & $1.27 \pm 1.28$ & $1.63 \pm 1.54$ & $<0.001$ & $<0.001$ \\
\hline Mitral disease (\%) & $28,263(75.8)$ & $13384(90.2)$ & $6142(78.9)$ & $8992(60.1)$ & $<0.001$ & $<0.001$ \\
\hline Tricuspid disease (\%) & $990(2.7)$ & $362(2.4)$ & $227(2.9)$ & $401(2.7)$ & 0.032 & 0.062 \\
\hline Endocarditis (\%) & $2050(5.5)$ & $250(1.7)$ & $592(7.6)$ & $1224(8.2)$ & $<0.001$ & $<0.001$ \\
\hline Intra Aortic Balloon Pump (\%) & $2043(5.5)$ & $635(4.3)$ & $1091(14)$ & $361(2.4)$ & $<0.001$ & $<0.001$ \\
\hline
\end{tabular}

*Comparison between patients undergoing mitral valve repair and replacement. †Comparison between all three groups (patients undergoing mitral valve repair, replacement, and those who did not receive surgery) 
of the Charlson Comorbidity and comorbidities as well as hospital level variables, such as hospital bed size, hospital region, and teaching status were included in the model. A $P$-value of less than 0.05 was considered significant. All analyses were performed using Stata IC 13 (Stata Corp, College Station, TX).

\section{RESULTS}

\section{Baseline clinical characteristics}

A total of 37,287 CTR cases were estimated in the US between 2000 and 2012. Among these patients, 14,833 $(39.8 \%)$ underwent mitral valve repair and $7780(20.9 \%)$

Table 2. Trends in patients with chordae tendineae rupture (CTR) (2000-2012)

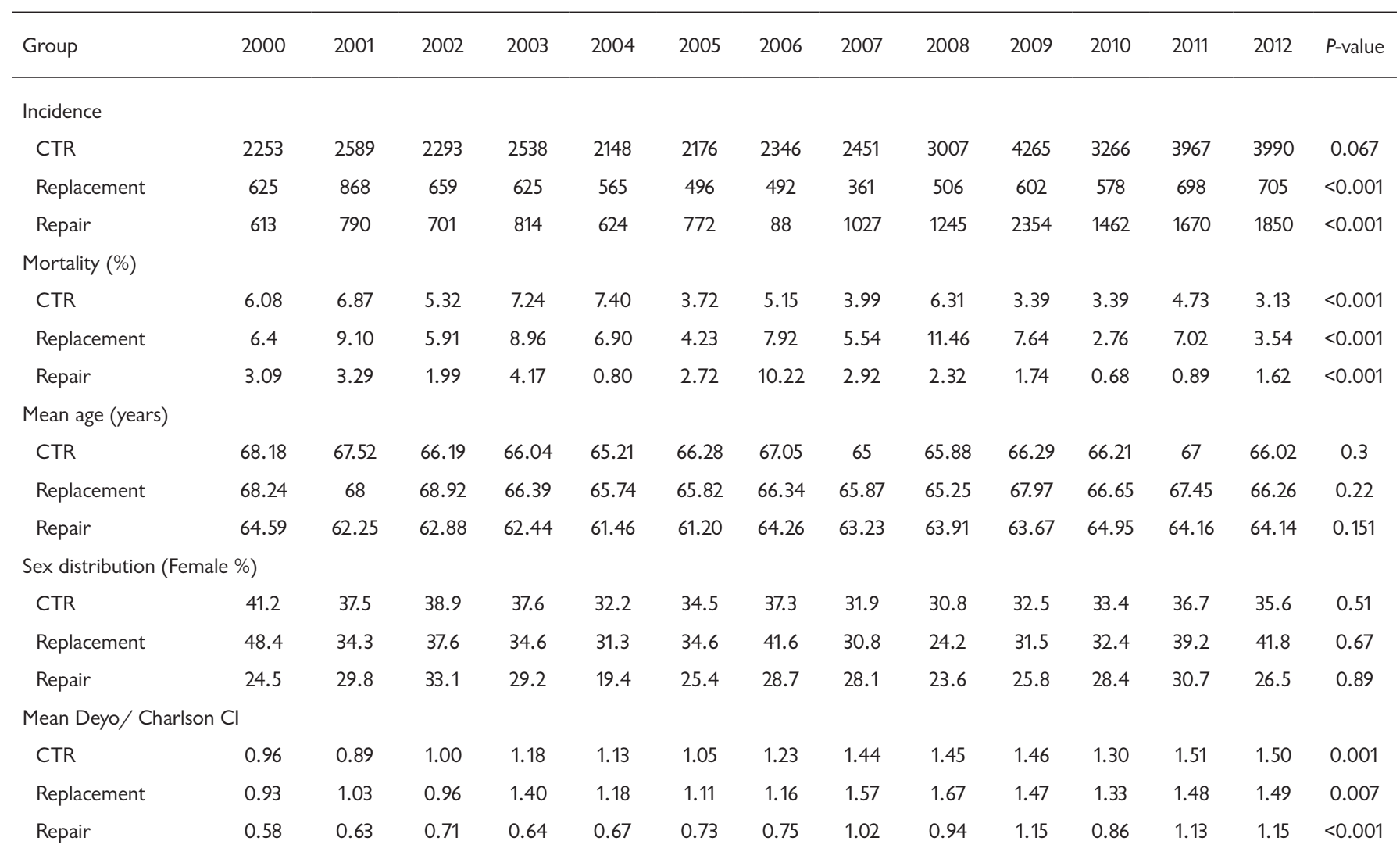

Median Length of Stay (days)

\begin{tabular}{|c|c|c|c|c|c|c|c|c|c|c|c|c|c|c|}
\hline CTR & 7 & 7 & 7 & 7 & 7 & 7 & 7 & 6 & 7 & 6 & 7 & 7 & 7 & 0.42 \\
\hline Replacement & 11 & 10 & 11 & 10 & 10 & 10 & 9 & 12 & 11 & 11 & 9 & 11 & 11 & 0.40 \\
\hline Repair & 7 & 6 & 7 & 7 & 7 & 7 & 7 & 7 & 7 & 6 & 6 & 7 & 6 & 0.22 \\
\hline
\end{tabular}

Cost (USD adjusted for inflation)

\begin{tabular}{|c|c|c|c|c|c|c|c|c|c|c|c|c|c|c|}
\hline CTR & 30614 & 31493 & 32095 & 32841 & 34473 & 36027 & 35399 & 38086 & 38336 & 36327 & 39511 & 43764 & 44288 & $<0.001$ \\
\hline Replacement & 48242 & 49556 & 47149 & 57809 & 57743 & 54226 & 58635 & 72553 & 63922 & 65358 & 62278 & 73485 & 73234 & $<0.001$ \\
\hline Repair & 38148 & 39120 & 33147 & 38972 & 42892 & 39065 & 39822 & 47636 & 43712 & 38209 & 42351 & 50533 & 48432 & $<0.001$ \\
\hline \multicolumn{15}{|l|}{ IABP (\%) } \\
\hline CTR & 2.9 & 4 & 5.9 & 5.5 & 7 & 7.4 & 6.4 & 4.5 & 7.2 & 5.8 & 4.9 & 4.4 & 5.8 & 0.62 \\
\hline Replacement & 8.7 & 8 & 12.5 & 13.6 & 15.3 & 18 & 16.7 & 11.9 & 24.6 & 20.5 & 12.8 & 11 & 14.2 & 0.20 \\
\hline Repair & 1.6 & 3.2 & 4.8 & 4.2 & 6 & 6.1 & 5.3 & 6.1 & 5.3 & 3.5 & 3.4 & 4.3 & 3.5 & 0.72 \\
\hline
\end{tabular}

$\operatorname{LVAD}(\%)$

CTR

Replacement

Repair

\begin{tabular}{|c|c|c|c|c|c|c|c|c|c|c|c|c|c|}
\hline 0 & 0 & 0 & 0 & 0 & 0 & 0 & 0 & 0 & 0 & 0 & 0.35 & 0.12 & 0.03 \\
\hline 0 & 0 & 0 & 0 & 0 & 0 & 0 & 0 & 0 & 0 & 0 & 2 & 0.7 & 0.03 \\
\hline 0 & 0 & 0 & 0 & 0 & 0 & 0 & 0 & 0 & 0 & 0 & 0 & 0 & 0 \\
\hline
\end{tabular}


underwent mitral valve replacement. Patient clinical characteristics and associated comorbidities are shown in Table 1. The mean age of patients with CTR was $66.37 \pm 15.25$ years and most patients were male $(64.8 \%)$.

Approximately $39 \%$ of patients with CTR did not receive any surgical intervention. Overall, patients in this group were found to be significantly older and have a higher burden of comorbidities (Table 1). Particularly, the rates of chronic valvular disease, chronic renal insufficiency, and congestive heart failure were drastically higher among patients who did not receive any surgical intervention $(P<0.001)$.

Compared to patients who underwent mitral valve replacement, patients with mitral valve repair were younger and more often male; were less likely to have congestive heart failure, diabetes, chronic renal failure, chronic liver disease, chronic valvular disease, atrial fibrillation, acute myocardial infarction, tricuspid disease, or endocarditis; had lower Deyo/Charson comorbidity index; and were more likely to have been diagnosed with hypertension and mitral disease (for all $P<0.001$ ). Total length of hospital stay was shorter in recipients of mitral valve repair compared to patients who underwent mitral valve replacement ( 7 versus 10 days respectively, $P<0.001$ ).

Overall, $19(0.1 \%)$ patients with CTR received an LVAD, and $2043(5.5 \%)$ received an IABP as a bridge to surgery. The percentage of patients receiving LVADs and IABPs was higher in patients who underwent mitral valve replacement compared to mitral valve repair $(0.2 \%$ versus $0 \%, P<0.001$ and $14 \%$ versus $4.3 \%, P<0.001$, respectively).

\section{Trends in patients with CTR}

No uniform trend was identified in the incidence of CTR from 2000 to $2012(P=0.067)$. While the mean age and sex distribution of patients with CTR did not change significantly from 2000 to 2012 (Table 2), the Deyo/Charlson comorbidity index showed a steady increase, from 0.96 to 1.52 , with an overall $58.3 \%$ increase in these patients over the study period. This upsurge was observed in both groups of patients

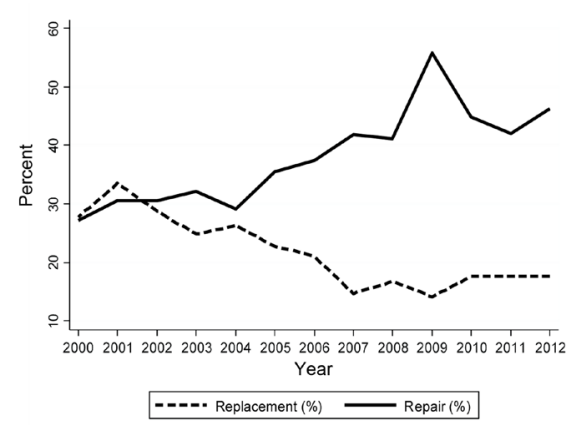

Figure 1. Trends in mitral valve repair and mitral valve replacement in patients with CTR (2000-2012). undergoing mitral valve repair and mitral valve replacement ( 0.58 to 1.15 , and 0.93 to 1.48 , respectively) (Table 2 ). Also, as shown in Figure 1, the rate of mitral valve repair increased significantly (by $19.2 \%, P<0.001$ ) with concomitant decrease in the incidence of mitral valve replacements (by $10.1 \%, P<$ $0.001)$ in these patients. There was no significant trend in the use of IABPs in patients undergoing either type of surgery during the study period (Table 2). LVADs were used only in patients undergoing mitral valve replacement in 2011 and 2012, and the overall numbers were very low.

\section{In-Hospital Mortality}

Overall, 1839 (4.9\%) patients with CTR died during admission, including $284(1.9 \%)$ of patients that underwent mitral valve repair and $525(6.8 \%)$ patients with mitral valve replacement. The mortality rate was highest among patients who did not receive any surgical intervention $(7.2 \%$, $P<0.001)$. Mortality was higher in patients who underwent mitral valve replacement when compared to patients that underwent repair $(6.8 \%$ versus $1.9 \%, P<0.001)$. However, as shown in Figure 2, the overall in-hospital mortality decreased by $3 \%$ among all patients with CTR from 2000 to 2012. This decrease in mortality rate was consistent for both groups of patients undergoing mitral valve repair and replacement (by $1.6 \%$ and $2.9 \%$, respectively) (Table 2). After multivariate adjustment for patient and hospital characteristics (Table 3), patients undergoing mitral valve replacement had greater odds of in-hospital death when compared to patients who underwent mitral valve repair (OR, 3.27, 95\% CI, 2.04-5.13, $P<0.001$ ) (Table 4).

\section{Predictors of Mortality}

Table 4 demonstrates the independent factors associated with mortality in patients with CTR. After multivariable adjustment, age, congestive heart failure, acute myocardial infarction, higher CCI, and use of IABP were found to be independently associated with greater odds of in-hospital

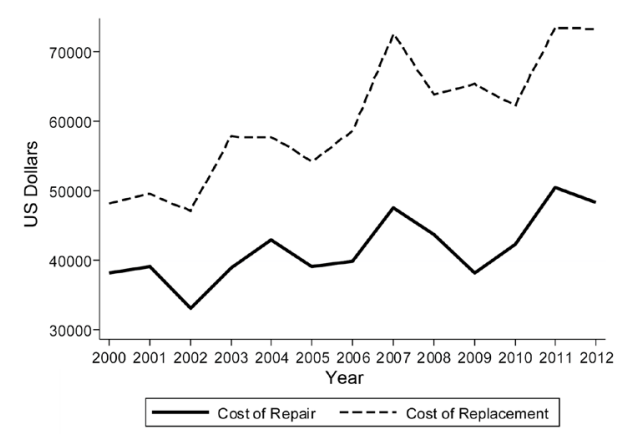

Figure 2. Trends in inflation adjusted cost of hospitalization in patients with CTR undergoing mitral valve repair and mitral valve replacement (2000-2012). 
mortality. Conversely, hypertension and valve repair were associated with lower odds of mortality in patients with ruptured chordae tendineae.

\section{In-Hospital Costs and Length of Stay}

Overall cost (adjusted for inflation) for patients with CTR has increased significantly from 2000 to 2012 (\$30,614 in 2000 versus $\$ 44,288$ in $2012, P<0.001$ ) (Table 2). Interestingly, the overall length of hospitalization did not exhibit any significant changes over the study period $(P=0.22)$. Similar results were observed in both groups of patients undergoing mitral valve repair and mitral valve replacement, with increases in the total in-hospital cost of care with no significant change in length of stay. (Table 2, Figure 2).

Using multivariable linear regression, mitral valve repair was shown to be significantly associated with lower total hospital costs compared with mitral valve replacement (Table 5). The strongest predictor of increased hospital cost was congestive heart failure followed by myocardial infarction. Other factors

Table 3. List of Parameters included in the multivariate logistic regression analysis

\begin{tabular}{l} 
Variable \\
\hline Age \\
Mortality \\
Gender \\
Year of procedure \\
Type of procedure \\
History of Congestive Heart Failure \\
History of Diabetes Mellitus \\
History of Hypertension \\
History of liver disease \\
History of Obesity \\
History of Myocardial Infarction \\
History of Mitral Disease \\
History of Tricuspid Disease \\
History of Endocarditis \\
History of Pulmonary Edema \\
History of Renal Failure \\
History of Chronic Obstructive Pulmonary Disease \\
History of Peripheral vascular Disease \\
History of Atrial Fibrillation \\
Hospital Size \\
Hospital Teaching Status \\
Hospital Region \\
Deyo score \\
History of Intra Aortic Balloon Pump \\
History of Left Ventricular Assist Device \\
\end{tabular}

associated with higher in-hospital costs included increasing age, female gender, chronic renal failure, and obesity, increasing in the CCI and medium and large hospital sizes.

\section{DISCUSSION}

\section{Study Findings}

In the present study we evaluated trends, mortality, and costs of mitral valve repair and mitral valve replacement among patients with ruptured chordae tendineae in the US between the years of 2000 and 2012. Analysis of over 37,000 patients revealed that mitral valve repair was associated with lower in-hospital mortality, shorter length of stay, and lower

Table 4: Independent Predictors of Mortality Among Patients with Rupture of Chordae Tendineae (2000-2012)

\begin{tabular}{lccc}
\hline Predictor of Mortality & Odds Ratio & $95 \% \mathrm{Cl}$ & P-value \\
\hline Age (Increasing, per y) & 1.04 & $1.03-1.06$ & $<0.001$ \\
Valve Repair & 0.35 & $0.19-0.63$ & 0.001 \\
Congestive Heart Failure & 2.08 & $1.19-3.64$ & 0.01 \\
Hypertension & 0.52 & $0.34-0.83$ & 0.005 \\
Acute Myocardial Infarction & 3.58 & $2.10-6.11$ & $<0.001$ \\
Deyo Comorbidity Index & 1.23 & $1.07-1.41$ & 0.003 \\
Intra Aortic Balloon Pump & 4.81 & $2.71-8.55$ & $<0.001$
\end{tabular}

Table 5. Independent Predictors of Cost of Hospitalization Among Patients with Rupture of Chordae Tendineae (20002012)

\begin{tabular}{lcc}
\hline Covariates & Coefficient (95\% Cl) & P-value \\
\hline Mitral Valve Repair & $-15693(-18749-12638)$ & $<0.001$ \\
Age (increasing, per y) & $235(1250-345)$ & $<0.001$ \\
Female Gender & $3135(282-6034)$ & 0.031 \\
Congestive Heart Failure & $55,358(44,726-65992)$ & $<0.001$ \\
Diabetes Mellitus & $-3598(-8322-1125)$ & 0.135 \\
Hypertension & $-3927(-6590-1263)$ & 0.004 \\
Chronic renal failure & $8121(2944-13299)$ & 0.002 \\
Chronic Liver Disease & $6308(-6701-19319)$ & 0.342 \\
Obesity & $6765(1784-11746)$ & 0.008 \\
Peripheral Vascular Disease & $-3000(-8330-2328)$ & 0.270 \\
Atrial Fibrillation & $1152(-1497-3802)$ & 0.394 \\
Myocardial Infarction & $32,416(25,021-39,810)$ & $<0.001$ \\
Deyo & $2218(930-3505)$ & 0.001 \\
Hospital Size & $8213(2435-13992)$ & 0.005 \\
Small & & $<0.001$ \\
Medium & $10207(3595-16819)$ & 0.002 \\
Large & &
\end{tabular}


in-hospital cost of care in patients with CTR. In-hospital mortality was also significantly decreased in patients with CTR over the same period. This trend was observed in both groups of patients undergoing mitral valve repair and replacement. Finally, the percentage of patients undergoing mitral valve repair increased steadily over this period with a concomitant decrease in the fraction of patients that underwent mitral valve replacement.

\section{Study Implications}

While MVP has become increasingly performed in a wide range of patient populations over the past decade [Badhwar 2012], few studies have reported its applicability among patients with CTR. The results of our study support mitral valve repair rather than replacement for patients with CTR. Although patients undergoing MVR had a higher burden of comorbidities in the present study, adjusted multivariate analysis demonstrated lower mortality in patients undergoing repair as compared to patients undergoing replacement. The benefit of mitral valve repair also applies to cost outcomes. Similar results were obtained in prior studies showing lower average inpatient costs for mitral valve repair as compared to replacement in other mitral valve disorders [Vassileva 2012; Kilic 2015].

\section{Trends}

While no significant changes were observed in the incidence of CTR, or in the age or gender distribution of patients, a sustained increase in the severity of comorbidity index was observed. This may be a true increase that reflects the overall increase in prevalence of diabetes, cardiovascular disease, acquired immunodeficiency syndrome, and other chronic conditions [Global Burden of Disease Study 2013 Collaborators 2015; Leon 2015] or it may be a consequence of improved diagnostic techniques, resulting in more accurate reporting of comorbidities in these patients. The increase in the burden of comorbidities could also be partly responsible for the significant increase in the costs of in-hospital care for both groups of patients undergoing MVR or MVP in our study.

In addition, the trend of in-hospital mortality in patients with CTR decreased over time. This effect was observed in both groups of patients undergoing MVP and MVR. This could in part be explained by improvement in surgical techniques over time. Also, overall reduction in mortality might also be due to increasing employment of MVP over MVR over the years [Gammie 2009].

\section{Predictors of in-hospital mortality}

As expected, older age was associated with higher mortality risk, which was previously reported for both mitral valve repair [Triantafyllis 2015] and replacement surgeries [Samiei 2014]. Additionally, we identified correlation of several comorbidities with mortality risk in our multivariable analysis. Congestive heart failure, acute myocardial infarction, higher CCI, and intra aortic balloon pump use were shown to have the strongest correlation with mortality. The association of increased mortality with congestive heart failure is not surprising, since preoperative ejection fraction was previously shown to be the strongest predictor of survival in patients undergoing mitral valve surgery [Triantafyllis 2015]. Similarly, the strong association of acute myocardial infarction with mortality was expected. Mortality in acute mitral regurgitation is determined by the underlying etiology, reaching $55 \%$ in patients with acute myocardial infarction [Thompson 2000], while non-ischemic chordal rupture due to myxomatous degeneration, mitral valve prolapse, or endocarditis are associated with lower mortality rates [Dal-Bianco 2014]. This may be explained in part by the fact that acute mitral regurgitation may present with acute pulmonary edema due to a rapid increase in volume in the left atrium and pulmonary venous system, while in chronic mitral regurgitation there is adaptation of the left ventricle and left atrium, resulting in an asymptomatic period prior to presentation.

Although to date there have been no studies looking at the burden of co-morbidities in patients with CTR, CCI was shown to be an independent predictor of mortality in various cardiac disorders [Bhavnani 2013; Lu 2013; Mamas 2015].

In our study, MVP and hypertension were independently associated with decreased mortality. The benefits of MVP have been previously studied for patients with mitral regurgitation of all etiologies [Gammie 2009; Kilic 2015] including degenerative valvular disease [McNeely 2015]. Our study further supports employment of MVP rather than MVR for patients with CTR, as MVP was independently associated with improved survival. However, it should be noted that the population of patients receiving MVR were drastically different from the patient population who underwent MVP. This supports the concept that the decision to choose MVR over MVP in patients with CTR should be tailored to the individual patient's clinical and anatomic characteristics. Further studies that include analysis of the degree of mitral regurgitation, the severity and extent of the disease, are warranted to identify the best possible individualized treatment options for patients with CTR.

\section{Limitations}

Our study has several limitations. First, the NIS is an administrative database, which is predisposed to errors arising from coding inaccuracies. However, in previous studies it was shown that inaccuracies observed in this type of database would likely result in undercoding rather than overcoding [Al-Khatib 2005]. Another limitation of this analysis is that some variables that could potentially affect the results were not available in the registry, such as: the type of surgery performed; the type of prosthesis used; the degree of mitral regurgitation; the severity of the condition on presentation; the extent of the disease; the area of expertise of the operating surgeon; and institutional resources. Third, despite the fact that NIS is designed to account for variability in cost among hospitals by using cost-to-charge ratios, in order to accurately address the issue of inpatient costs, further breakdown of costs into fixed and variable, and intraoperative and postoperative, would be desired. Unfortunately, it is not possible to derive these from NIS database. Fourth, NIS reports in-hospital mortality but is unable to capture events that occur after hospital discharge. Moreover, the database excludes data collected 
after patient transfer to another hospital or patient readmission for re-operation. Finally, given the limited number of parameters included in NIS, the possibility of residual unaccounted confounding factors cannot be excluded in the present study. Therefore, the results of the current study should be interpreted in the context of these limitations.

\section{CONCLUSIONS}

Our study reports contemporary trends in a large number of patients with CTR in the United States. The results indicate that MVP is associated with lower mortality rates and costs compared to MVR. These results support utilization of MVP in patients with CTR when technically feasible.

\section{REFERENCES}

Al-Khatib SM, Lucas FL, Jollis JG, et al. 2005. The relation between patients' outcomes and the volume of cardioverter-defibrillator implantation procedures performed by physicians treating Medicare beneficiaries. J Am Coll Cardiol 46:1536-1540.

Badhwar V, Peterson ED, Jacobs JP, et al. 2012. Longitudinal outcome of isolated mitral repair in older patients: results from 14,604 procedures performed from 1991 to 2007. Ann Thorac Surg 94:1870-1877; discussion $1877-1879$.

Bhavnani SP, Coleman CI, Guertin D, et al. 2013. Evaluation of the Charlson comorbidity index to predict early mortality in implantable cardioverter defibrillator patients. Ann Noninvasive Electrocardiol $18: 379-388$

Dal-Bianco JP, Beaudoin J, Handschumacher MD, et al. 2014. Basic mechanisms of mitral regurgitation. Can J Cardiol 30:971-981.

Deyo RA, Cherkin DC, Ciol MA. 1992. Adapting a clinical comorbidity index for use with ICD-9-CM administrative databases. J Clin Epidemiol 45:613-619.

Gabbay U, Yosefy C. 2010. The underlying causes of chordae tendinae rupture: a systematic review. Int J Cardiol 143:113-118.

Gammie JS, Sheng S, Griffith BP, et al. 2009. Trends in mitral valve surgery in the United States: results from the Society of Thoracic Surgeons Adult Cardiac Surgery Database. Ann Thorac Surg 87:1431-1437; discussion 1437-1439.

Global Burden of Disease Study 2013 Collaborators. 2015. Global, regional, and national incidence, prevalence, and years lived with disability for 301 acute and chronic diseases and injuries in 188 countries, 1990-2013: a systematic analysis for the Global Burden of Disease Study 2013. Lancet 386:743-800.

Kazui T, Kawazoe K. 2004. Acute mitral valve insufficiency caused by chordae rupture. Kyobu geka 57:656-662.

Kilic A, Grimm JC, Magruder JT, et al. 2015. Trends, clinical outcomes, and cost implications of mitral valve repair versus replacement, concomitant with aortic valve replacement. J Thorac Cardiovasc Surg 149:1614-1619.

Leon BM, Maddox TM. 2015. Diabetes and cardiovascular disease: Epidemiology, biological mechanisms, treatment recommendations and future research. World J Diabetes 6:1246-1258.

Lu KJ, Kearney LG, Ord M, et al. 2013. Age adjusted Charlson Comorbidity Index is an independent predictor of mortality over long-term follow-up in infective endocarditis. Int J Cardiol 168:5243-5248.

Mamas MA, Fath-Ordoubadi F, Danzi GB, et al. 2015. Prevalence and Impact of Co-morbidity Burden as Defined by the Charlson Co-morbidity Index on 30-Day and 1- and 5-Year Outcomes After Coronary Stent Implantation (from the Nobori-2 Study). Am J Cardiol 116:364-371.

McNeely CA, Vassileva CM. 2015. Long-term outcomes of mitral valve repair versus replacement for degenerative disease: a systematic review. Curr Cardiol Rev 11:157-162.

Rankin JS, Orozco RE, Addai TR, et al. 2004. Several new considerations in mitral valve repair. J Heart Valve Dis 13:399-409.

Samiei N, Hakimi MR, Mirmesdagh Y, et al. 2014. Surgical outcomes of heart valves replacement: A study of tertiary specialied cardiac center. ARYA Atheroscler 10:233-237

Steiner C, Elixhauser A, Schnaier J. 2002. The healthcare cost and utilization project: an overview. Eff Clin Pract 5:143-151.

Thompson CR, Buller CE, Sleeper LA, et al. 2000. Cardiogenic shock due to acute severe mitral regurgitation complicating acute myocardial infarction: a report from the SHOCK Trial Registry. SHould we use emergently revascularize Occluded Coronaries in cardiogenic shocK? J Am Coll Cardiol 36(3 Suppl A):1104-1109.

Triantafyllis AS, Kortlandt F, Bakker AL, et al. 2016. Long-term survival and preprocedural predictors of mortality in high surgical risk patients undergoing percutaneous mitral valve repair. Catheter Cardiovasc Interv $87: 467-75$

Vassileva CM, Shabosky J, Boley T, et al. 2012. Cost analysis of isolated mitral valve surgery in the United States. Ann Thorac Surg 94:1429-1436. 